\title{
Edema de membro inferior secundário a exérese de veia safena magna para utilização como enxerto na revascularização do miocárdio
}

\author{
Lower limb edema after great saphenous vein harvesting to be used \\ as graft in myocardial revascularization
}

\section{Cleusa Ema Quilici Belczak ${ }^{1}$, José Maria Pereira de Godoy², Rubiana Neves Ramos³, André Luiz Tyszka ${ }^{4}$, Sergio Quilici Belczak ${ }^{5}$, Roberto Augusto Caffaro ${ }^{6}$}

\section{Resumo}

Contexto: A revascularização do miocárdio utilizando-se a veia safena magna ainda é procedimento cirúrgico bastante realizado na atualidade. $\mathrm{O}$ edema que surge no membro inferior operado causa grande desconforto e necessita ser melhor estudado.

Objetivos: Caracterizar o edema de membro inferior secundário a exérese da veia safena magna pela técnica de incisões escalonadas para sua utilização como enxerto venoso na revascularização do miocárdio.

Métodos: Foram selecionados aleatoriamente 44 indivíduos submetidos a exérese de veia safena magna para revascularização miocárdica há mais de 3 meses. Excluíram-se fatores que pudessem interferir na formação de edema dos membros inferiores. Foram avaliados por volumetria e perimetria maleolares ambos os membros inferiores. Considerou-se como presença de edema significativo a diferença de volume maior que $50 \mathrm{~mL}$ e maior de $2 \mathrm{~cm}$ em relação ao membro não-operado. Para a análise estatística foram empregados o teste do qui-quadrado, teste exato de Fisher, teste $t$ de Student e o teste de McNemar. O nível de significância adotado foi de 5\% $(\alpha=$ $0,05)$.

Resultados: Encontraram-se diferenças estatisticamente significativas $(\mathrm{p}<0,05)$ entre os membros operados e os não-operados, sendo $56,8 \%$ com volume maior que $50 \mathrm{~mL}$ e $31,9 \%$ com perímetro maleolar maior que $2 \mathrm{~cm}$. Não se encontrou associação entre a presença do edema e características da amostra ou da cirurgia e de intercorrências clínicas perioperatórias ou tardias.

Conclusões: Pacientes submetidos a ressecções de veia safena magna para sua utilização como ponte coronariana poderão apresentar edema no membro associado.

Palavras-chave: Edema, revascularização miocárdica, diagnóstico, complicações.

\section{Abstract}

Background: Myocardial revascularization using the great saphenous vein is still a very common surgical procedure. The edema that occurs in the operated leg causes much discomfort and requires further studies.

Objectives: To describe lower limb edema secondary to great saphenous vein harvesting using the bridge technique for use as venous graft in myocardial revascularization.

Methods: Forty-four individuals previously submitted to great saphenous vein harvesting for myocardial revascularization more than 3 months before were randomly selected. Patients with factors that might interfere with formation of lower limb edema were excluded. Both operated and non-operated legs were evaluated by volumetry and perimetry of the malleolar region. Differences greater than 50 $\mathrm{mL}$ or higher than $2 \mathrm{~cm}$ between the operated and the contralateral leg were considered as presence of significant edema. Chi-square, Fisher's, McNemar's and Student's t tests were used for statistical analysis. Significance level was set at $5 \%(\alpha=0.05)$.

Results: Statistically significant differences $(p<0.05)$ were found between operated and contralateral legs, with $56.8 \%$ of the individuals having a difference in volume greater than $50 \mathrm{~mL}$ and $31.9 \%$ having a difference in malleolar region perimeter greater than $2 \mathrm{~cm}$. There was no association between presence of edema and sample characteristics, surgery, perioperative or late clinical complications.

Conclusions: Patients submitted to resection of the great saphenous vein for coronary artery bypass grafting may evolve with edema of the operated leg.

Keywords: Edema, myocardial revascularization, diagnosis, complications.

1. Doutoranda, Cirurgia Geral, Faculdade de Ciências Médicas, Irmandade da Santa Casa de Misericórdia de São Paulo, São Paulo, SP. Professora, Curso de Pós-Graduação em Reabilitação Linfovenosa, Faculdade de Medicina de São José do Rio Preto (FAMERP), São José do Rio Preto, SP.

2. Doutor. Livre-docente. Professor adjunto, Cardiologia e Cirurgia Cardiovascular, Departamento de Cirurgia, FAMERP, São José do Rio Preto, SP. Pesquisador, CNPq.

3. Fisioterapeuta, Centro Vascular João Belczak, Maringá, PR. Pós-graduação, Morfologia e Métodos de Ensino e Pesquisa, Universidade Estadual de Maringá (UEM), Maringá, PR.

4. Cirurgião torácico e cardiovascular. Ex-residente, Hospital de Clínicas, Universidade Federal do Paraná (UFPR), Curitiba, PR.

5. Residente, Cirurgia Vascular, Hospital das Clínicas, Universidade de São Paulo (USP), São Paulo, SP.

6. Doutor. Professor adjunto, Disciplina de Cirurgia Vascular, Faculdade de Ciências Médicas, Irmandade da Santa Casa de Misericórdia de São Paulo, São Paulo, SP.

Não foram declarados conflitos de interesse associados à publicação deste artigo.

Artigo submetido em 16.09.08, aceito em 24.11.08

J Vasc Bras. 2009;8(1):43-47.

Copyright $\odot 2009$ by Sociedade Brasileira de Angiologia e de Cirurgia Vascular 


\section{Introdução}

Apesar de o avanço da cirurgia endovascular e da crescente realização de angioplastias com ou sem a colocação de stents, a cirurgia convencional para revascularização do miocárdio por meio de enxerto venoso utilizando-se a veia safena magna continua a ser executada em ampla escala em todo o planeta. Estima-se a realização de aproximadamente 800.000 de tais intervenções por ano ${ }^{1-3}$. As complicações que sobrevêm no membro inferior operado têm sido negligenciadas e certamente ainda não foram devidamente estudadas ${ }^{4-6}$.

Apesar de os cuidados que visam simplificar o procedimento reduzirem o trauma operatório e tornarem as cicatrizes esteticamente menos visíveis, a longo prazo a maioria desses pacientes apresentam, no membro operado, intercorrências clínicas que causam desconforto e levam à busca de tratamento especializado ${ }^{3}$. Na prática angiológica cotidiana, tem-se constatado que o edema do membro operado, cuja manifestação nem sempre traz queixa espontânea, apresenta-se com frequência bem mais alta do que aquele mencionado na literatura ${ }^{7}$. Alguns indivíduos costumam, inclusive, desenvolver episódios de linfangite e ou erisipelas recorrentes, o que, seguramente, perpetua e agrava o quadro edematoso $0^{8-10}$.

O objetivo deste trabalho é estudar o edema secundário no membro inferior submetido a exérese da veia safena magna por técnica escalonada para sua utilização como enxerto venoso na revascularização do miocárdio.

\section{Métodos}

Foram selecionados aleatoriamente, em estudo observacional e transversal, 44 pacientes submetidos a revascularização do miocárdio com utilização da veia safena magna para enxerto venoso extraída pela técnica de incisões escalonadas há mais de 3 meses, da Clínica de Cirurgia Cardíaca de Maringá (PR). Os participantes assinaram um termo de consentimento após terem sido informados sobre o objetivo deste estudo, que foi previamente submetido à apreciação e posterior aprovação pelo Comitê de Ética da Faculdade de Ciências Médicas da Santa Casa de São Paulo.
Como critério de inclusão, foram selecionados os pacientes que haviam sido submetidos a revascularização do miocárdio utilizando-se a veia safena magna. Por outro lado, foram excluídos aqueles:

- cuja incisão fosse contínua e longa desde a região inguinal até o maléolo;

- que tivessem retirado a veia safena magna de ambos os lados ou que tivessem sido submetidos a safenectomia prévia do membro contralateral, quer para tratamento de hipertensão venosa superficial por varizes, quer para a sua utilização em revascularização arterial distal;

- que fossem portadores de edema de outra etiologia, como causas sistêmicas (renais, tireoideas, hepáticas, discrásicas, etc.) ou locais (trauma, afecções reumáticas e/ou ortopédicas), ou ainda com insuficiência venosa nos membros inferiores, representada por varizes exuberantes com ou sem alterações tróficas;

- que estivessem na vigência de medicação diurética ou hormonal;

- que apresentassem ulcerações ou lesões de pele com ou sem solução de continuidade nos membros inferiores;

- com quadro de isquemia dos membros inferiores;

- com idade superior a 75 anos.

Todos os pacientes foram submetidos no período da manhã, sempre pela mesma fisioterapeuta, a medições perimétricas a $1 \mathrm{~cm}$ do tornozelo, considerando variações maiores que $2 \mathrm{~cm}$, e a volumetria com a utilização da técnica de deslocamento de água, considerando variações maiores que $50 \mathrm{~mL}$. Avaliou-se a associação do edema com diabetes, infecção, tempo de cicatrização ou, ainda, com características cirúrgicas, como número e comprimento das incisões e o posterior aparecimento de parestesias.

Para a análise estatística foram empregados o teste do qui-quadrado, teste exato de Fisher, teste $t$ de Student e o teste de McNemar, considerando erro alfa de $5 \%$. 
Tabela 1 - Variações volumétricas e perimétricas do estudo

\begin{tabular}{lccc}
\hline & Membro operado & $\begin{array}{c}\text { Membro } \\
\text { não-operado }\end{array}$ & $\begin{array}{c}\text { Diferença } \\
\text { (operado versus não-operado) }\end{array}$ \\
\hline Perímetro (cm) & & & \\
Média \pm DP & $24,5 \pm 2,6$ & $23,2 \pm 2,4$ & $1,3 \pm 1,5$ \\
Mediana & 24 & 23 & 1,0 \\
Mínimo-máximo & $20-33$ & $19-29$ & -1 a 6 \\
IC95\% para a diferença & & & 0,$76 ; 1,66$ \\
Volume (mL) & $3.147,2 \pm 470,0$ & $3.069,3 \pm 424,2$ & $77,8 \pm 128,2$ \\
Média \pm DP & 3.040 & 2.985 & 50 \\
Mediana & $2.600-4.490$ & $2.600-3.980$ & -180 a 570 \\
Mínimo-máximo & & & 38,$9 ; 116,8$ \\
IC95\% para a diferença & 44 & 44 & 44 \\
Total de pacientes & & & 0,001 \\
\hline
\end{tabular}

* Teste $t$ de Student pareado.

DP = desvio padrão; IC $95 \%$ = intervalo de confiança de $95 \%$.

\section{Resultados}

Todos os indivíduos da amostra eram destros e nenhum era obeso mórbido. Do total, 32 eram homens e 12, mulheres, e suas idades variaram de 47 a 75 anos. Quanto ao grupo étnico, 36 indivíduos eram brancos, três negros, um amarelo e quatro eram pardos (mulatos).

Somente em cinco pacientes a cirurgia do membro foi feita no lado direito; nos demais 39 , todas foram efetuadas do lado esquerdo por preferência técnica. Dezenove pacientes eram diabéticos e duas das mulheres tinham lipedema familiar de membros inferiores.

Quanto ao tempo decorrido entre a cirurgia e o dia da avaliação, os indivíduos foram divididos em três grupos: grupo I (até 1 ano), com oito indivíduos (18,2\%); grupo II (de 1 a 5 anos), com 23 indivíduos (52,3\%); e grupo III (mais de 5 anos), com 13 indivíduos (29,5\%).

Dos 44 pacientes avaliados, 24 referiram o surgimento e a sensação de edema no membro operado, cujo início ocorrera entre 30 dias e 6 meses após o ato cirúrgico. Com relação às medidas, 33 pacientes $(75 \%)$ evidenciaram perímetros maiores nos membros operados, sete $(15,9 \%)$ mostraram perímetros iguais e quatro $(9,1 \%)$ relataram perímetros menores. Na volumetria, encontraram-se $35(79,5 \%)$ indivíduos com volumes maiores no membro operado, dois $(4,6 \%)$ com volumes iguais e sete $(15,9 \%)$ com volumes menores. Em nove $(20,5 \%)$ dos pacientes que se queixaram de edema no membro operado, não se encontrou volume significativo que justificasse a presença de edema, e em quatro $(9,1 \%)$ foram encontrados volumes aumentados no membro não-operado, conforme a Tabela 1.

As maiores diferenças de volume entre os membros operados e não-operados foram encontradas nos quatro pacientes que apresentaram episódio de erisipela no pós-operatório tardio, com uma média de $320 \mathrm{~mL}$ a favor do membro operado. Não houve relação entre presença de edema e outras características da amostra, como o diabetes, infecção e tempo de cicatrização ou ainda com as características cirúrgicas, como número e comprimento das incisões e o posterior aparecimento de parestesias.

\section{Discussão}

O presente estudo mostra que a retirada da veia safena para confecção das pontes coronarianas está associada ao desenvolvimento do edema pós-operatório no membro submetido ao procedimento. Outro dado analisado foi a sensação de edema em $54,5 \%$, o que não foi coincidente em 20,5\%, mostrando que muitas vezes o paciente tem sensação de edema, mas não o apresenta 
de fato. Entretanto, não se observou relação com diabetes, infecção, parestesias, tempo de cicatrização e/ou comprimento das incisões.

Sabendo-se que, comumente, o lado dominante costuma apresentar-se maior ${ }^{11,12}$, vale lembrar que todos os pacientes de nossa amostra eram destros $(100 \%)$ e foram operados, na sua grande maioria, no lado esquerdo $(88,6 \%)$. Assim, o fato de encontrarmos um aumento significativo de volume no lado operado confirma a presença real de edema.

Terada et al. ${ }^{13}$ avaliaram a origem do edema pósretirada da veia safena magna, supondo ser esse de origem venosa, e estudaram, por Eco-Doppler colorido, o sistema venoso superficial e profundo dos membros edematosos operados. Os autores não encontraram nenhuma anomalia anatômica ou funcional que justificasse a formação desse edema. Por outro lado, sabe-se que as lesões linfáticas, que podem ocorrer quando da dissecção e exérese da veia safena magna em função da íntima relação que guardam entre si os sistemas venosos e linfáticos do ponto de vista embriológico, anatômico e funcional, explicariam a origem do edema como fator consequente da ruptura das vertentes linfáticas paralelas entre si, bem como todo o trajeto venoso troncular magno.

Estudo avaliando 302 pacientes em condições semelhantes às de nossa mostra encontrou história de erisipela em 21 deles, sendo que esses pacientes apresentaram os maiores volumes, sugerindo envolvimento linfático no edema ${ }^{9}$, o que coincide com a nossa pesquisa. Há evidência na literatura de que a erisipela de repetição é uma condição associada ao linfedema ${ }^{14}$. Estes dados falam a favor de uma forte interferência do sistema linfático no edema pós-remoção da veia safena, o que em futuro próximo certamente poderá ser melhor avaliada e quiçá confirmada por estudos linfocintilográficos.

Um simples exame, como a volumetria, pode ser usado na avaliação destes pacientes e pode, ainda, sugerir a provável presença de linfedema clínico ou subclínico no membro associado. Uma das limitações de nosso estudo foi a falta de confirmação do linfedema por meio diagnóstico mais específico, como, por exemplo, a linfocintilografia.
Pelo exposto, pode-se afirmar que a presença do edema serve como alerta da presença e desenvolvimento de provável linfedema secundário, o que justifica cuidados profiláticos a serem tomados para prevenção de infecções que agravariam o quadro edematoso de forma irreversível.

\section{Conclusão}

Pacientes submetidos a ressecções de veia safena magna para sua utilização em ponte coronariana poderão apresentar edema secundário no membro associado, cuja origem provavelmente se deva ao trauma linfático ocorrido no setor.

\section{Referências}

1. De Milto L, Costello AM. Coronary artery bypass graft surgery. Gale Encyclopedia of Surgery. Gale: The Gale Group; 2004. http://www.healthline.com/galecontent/coronary-arterybypass-graft-surgery-1. Acessado: 29/02/2008.

2. Favoloro RG. Critical analysis of coronary bypass graft surgery: a 30 year journey. J Am Coll Cardiol. 1998;31(4Suppl B):1B-63B.

3. Tyszka AL, Fucuda LS, Tormena EB, Campos ACL. Obtenção da veia safena magna através de acesso minimamente invasivo para revascularizações miocárdicas. Rev Bras Cir Cardiovasc. 2001;16:105-13.

4. Bruxton B, Acar C, Suma H, et al. Conduits. In: Bruxton B, Frazier $\mathrm{OH}$, Westaby S. Ischemic heart disease surgical management. London: Mosby; 1999. p. 139-77.

5. Reid R, Simcock JW, Chisholm L, Dobbs B, Frizelle FA. Postdischarge clean wound infections: incidence underestimated and risk factors overemphasized. ANZ J Surg. 2002;72:339-43.

6. Garland R, Frizelle FA, Dobbs BR, Singh H. A retrospective audit long-term lower limb complications following leg vein harvesting for coronary artery bypass grafting. Eur J CardioThoracic Surg. 2003;23:950-5.

7. Lavee J, Schneiderman J, Yorav S, Shewach-Millet M, Adar R. Complications of saphenous vein harvesting following coronary artery bypass surgery. J Cardiovasc Surg (Torino). 1989;30:989-91.

8. Schoppelrey HP, Breit R. [Erysipelas after leg vein harvesting for aortocoronary bypass operation]. Hautarzt. 1996;47:909-12.

9. Dan M, Heller K, Shapira I, Vidne B, Shibolet S. Incidence of erysipelas following venectomy for coronary artery. Infection. 1987; 15:107-8.

10. Eckel L. Die vene als transplantat: aortokoronarer und peripherer venenbypass. Z Kardiol. 1991;80:73-7.

11. Belczak CEQ, Godoy JMP, Seidel AC, Silva JA, Cavalheri Jr G, Belczak SQ. Influência da atividade diária na volumetria dos membros inferiores medida por perimetria e por pletismografia de água. J Vasc Bras. 2004;3:304-10. 
12. Nilsson S, Haugen GB. Volumetry in evaluation of swelling in the ankle and foot. J Oslo City Hosp. 1981;31:11-5.

13. Terada Y, Fukuda S, Tohda E, Kigawa I, Wanibuchi Y, Mitsui T. Venous function and delayed leg swelling following saphenectomy in coronary artery bypass grafting. J Thorac Cardiovasc Surg. 1999;47:559-62.

14. de Godoy JM, de Godoy MF, Valente A, Camacho EL, Paiva EV. Lymphoscintigraphic evaluation in patients after erysipelas. Lymphology. 2000;33:177-80.
Correspondência:

Cleusa Ema Quilici Belczak

Centro Vascular João Belczak

Av. Tiradentes, 1081

CEP 87013-260 - Maringá, PR

E-mail: belczak@wnet.com.br

\title{
ATENÇÃO
}

\section{Registros de Ensaios Clínicos}

O Jornal Vascular Brasileiro apóia as políticas para registro de ensaios clínicos da Organização Mundial da Saúde (OMS) e do International Committee of Medical Journal Editors (ICNJE, www.icmje.org), reconhecendo a importância dessas iniciativas para o registro e a divulgação internacional de informação sobre estudos clínicos, em acesso aberto. De acordo com essa recomendação, artigos de pesquisas clínicas devem ser registrados em um Registro de Ensaios Clínicos validados pelos critérios estabelecidos pela OMS e ICMJE, cujos endereços estão disponíveis no site do ICMJE:

\author{
www.actr.org.au \\ www.clinicaltrials.gov \\ www.isctn.org \\ www.umin.ac.jp/ctr/index/htm \\ www.trialregister.nl
}

O número de identificação deverá ser registrado ao final do resumo

A partir de 2008, somente serão aceitos para publicação artigos que tenham recebido um número de identificação nesses registros. 